\title{
Cross-cultural adaptation and validation of a Quality of Life questionnaire: The Nasal Obstruc- tion Symptom Evaluation questionnaire*
}

\author{
Thiago F.P. Bezerra ${ }^{1}$, Francini G.M. Padua ${ }^{1}$, Renata R. de M. Pilan ${ }^{1}$, Michael G. \\ Stewart $^{2}$, Richard L. Voegels ${ }^{1}$ \\ Department of Otorhinolaryngology and Ophthalmology, School of Medicine, University of São Paulo, Sao \\ Paulo, Brazil \\ 2 Department of Otorhinolaryngology, Weill-Cornell Medical College, New York, USA
}

SUMMARY Background: The Nasal Obstruction Symptom Evaluation (NOSE) instrument is a diseasespecific questionnaire for assessing the outcome of an intervention in nasal obstruction in trials. This instrument is only available in the English language and cross-culturally valid questionnaires are very important for all research, including nasal obstruction. The aim of the current study was to reproduce the cross-cultural adaptation process for the NOSE questionnaire in the Portuguese language (NOSE-p).

Methodology: Cross-cultural adaptation and validation of the instrument were divided into two stages. Stage 1 involved four bilingual professionals, an expert committee and the author of the original instrument. In Stage 2, the NOSE-p was tested on 33 patients undergoing septoplasty for internal consistency, test-retest reliability, construct validity, discriminant validity, criterion validity, and response sensitivity.

Results: The cross-cultural adaptation process was completed and the NOSE-p was demonstrated to be a valid instrument with satisfactory construct validity. It showed an adequate internal consistency reliability and adequate test-retest reliability. It could discriminate between patients with and without nasal obstruction and it has a high response sensitivity to change.

Conclusions: The cross-cultural adaptation and validation process demonstrated to be valid and the NOSE-p proved to be applicable in Brazil.

Key words: nasal obstruction/diagnosis, quality of life, validation studies, outcome assessment (health care), health status indicators

\section{INTRODUCTION}

Nasal obstruction is defined as a discomfort manifested as a feeling of insufficient airflow through the nose. It may be a chronic condition with a great burden that impacts significantly on the quality of life of patients. The prevalence of nasal obstruction has been estimated at $26.7 \%$, in urban dwellers ${ }^{(1)}$. There are many causes of this condition, such as rhinitis, adenoid hypertrophy, among others. Nasal septal deviation is a very common cause of nasal obstruction and septoplasty is the definitive treatment of this condition.

There is no agreement on an accepted measurement tool for objective assessment of nasal obstruction after septoplasty (2). There were several prior studies with non-validated instruments to evaluate patients' subjective outcome after septoplasty ${ }^{(3-5)}$, until Stewart et al. reported the validation of NOSE ("Nasal Obstruction Symptom Evaluation Scale") in $2004^{(6)}$. Diseasespecific quality of life instruments are important ways to assess the impact of a disease or its treatment on patients.
The NOSE instrument is a disease-specific questionnaire for assessing the outcome of an intervention in nasal obstruction in trials ${ }^{(7)}$. It is structurally composed of five obstruction-related items, which address the severity of complaints that the patient has been experiencing over the past month with reference to each of the following: 1) 'nasal congestion or stuffiness;' 2) 'nasal blockage or obstruction;' 3) 'trouble breathing through my nose;' 4) 'trouble sleeping' and 5) 'Unable to get enough air through my nose during exercise or exertion.' All 5 items are scored using a 5-point Likert scale (not a problem, very mild problem, moderate problem, fairly bad problem, and severe problem). The instrument is then scaled to a total score of 0 to 100 by multiplying the raw score by 5 . Due to the wording of the items, a score of 0 means no problems with nasal obstruction and a score of 100 means the most severe problem possible with nasal obstruction ${ }^{(}$.

Increasingly, health service researchers use standardized questionnaires designed for producing data that can be compared across studies ${ }^{(8)}$. The cross-cultural adaptation 
of a health status self-administered questionnaire for use in a new country, culture, and/or language necessitates the use of a unique method, to reach equivalence between the original source and translated versions of a questionnaire. If measures are to be used across cultures, the items must not only be translated well linguistically, but also must be adapted culturally to keep the content validity of the instrument at a conceptual level across different cultures. Attention to this level of detail ensures increased confidence that the impact of a disease or its treatment is described in a similar consistent manner in multinational trials or outcome evaluations ${ }^{(9)}$. Using a previously validated and published questionnaire will still save time and resources, as opposed to developing an entirely new instrument in a new language ${ }^{(8)}$.

This is the first adaptation of NOSE into a non-English language. There is no Portuguese version of NOSE available at present and a rigorous process of cross-cultural adaptation and validation is therefore needed. The aim of this study is the cross-cultural adaptation and validation of the NOSE instrument into the Portuguese language.

\section{MATERIALS AND METHODS}

Set up of the study

This was a prospective cohort study conducted at Clinics Hospital, University of São Paulo, with Ethics committee approval. All patients agreed and signed consent forms.

\section{Cross-cultural adaptation process}

The cross-cultural adaptation process of the NOSE instrument was performed using standard techniques ${ }^{(8,9)}$. Two forward translations were made of NOSE (original questionnaire) from English to Portuguese by two bilingual translators whose mother tongue is Portuguese. One of the translators had a medical background and the other had a non-medical background. The two translators, a recording observer and an expert committee synthesized the results of the translations in a Portuguese version of NOSE. Working from this version of the questionnaire and totally blind to the original version, two translators with English as their mother tongue and without medical background translated the questionnaire back into the original language. The two translators, a recording observer and an expert committee synthesized the results of the translations in an English back-translated version. Documentation of all steps was submitted to the author of the original questionnaire for appraisal of the adaptation process, and the final version of the Portuguese questionnaire (NOSE-p) for testing was created.

\section{Subjects}

Patients were enrolled consecutively. The enrolment period was from 1st June 2008 until 31st December 2008. Inclusion criteria for the study were: a) Patients with nasal obstruction due to nasal septal deviation with or without inferior turbinate hypertrophy compatible with chronic nasal obstruction; b) symptoms lasting 12 months; c) persistent symptoms after a 8-week trial of medical management, including topical nasal steroids and oral antihistamine-decongestant combinations (for patients with concurrent allergic rhinitis); d) surgery indication for septoplasty; e) age of at least 18 years. Exclusion criteria were: a) sinonasal malignancy; b) radiation therapy to the head and neck; c) septoplasty performed with concurrent sinus surgery, rhinoplasty, or as an access to other sites; d) prior nasal surgery; f) chronic rhinosinusitis (using the EP3OS definition ${ }^{(7)}$ ); g) septal perforation; craniofacial syndrome; h) anterior nasal trauma or fracture; i) adenoid hypertrophy; j) sarcoidosis; k) granulomatosis of the nasal cavity; i) uncontrolled asthma; j) pregnancy.

An additional group of patients was collected as a convenience sample for between groups discrimination. Patients in the otolaryngology clinics of the Clinics Hospital of the University of Sao Paulo without rhinological complaints were asked to complete the NOSE-p Scale.

\section{Methods}

Patients completed the NOSE-p questionnaire about 7 days before septoplasty surgery and 90 days after surgery. About 15 days after this last consultation, patients completed the NOSE-p questionnaire again to assess test-retest reliability. This was calculated using the Goodman-Kruskal gamma coefficient.

\section{Statistical analyses}

Internal consistency reliability was estimated by calculating Cronbach's alpha, and inter-item and item-total correlations. Internal consistency reliability was considered acceptable if Cronbach's alpha was 0.70 or higher ${ }^{(6)}$.

Validity was confirmed by the face validity of the dualtranslation process. In addition, discriminant validity was estimated by comparing total scores between two groups: study patients and a sample of patients seen without rhinologic complaints. The Mann-Whitney U test was used.

Sensitivity was assessed by calculating the standardized response mean and effect size, at 90 days after surgical intervention; these values of which were compared with published standards. Values of approximately 0.2, 0.5 and 0.8 represent low, moderate and high sensitivity to change, respectively ${ }^{(6)}$.

The sample size was calculated using the correlation coefficient of 0.40 as an outcome. An alpha value of 0.05 was considered statistically significant for all statistic tests. Data analysis was carried out using SPSS version 10.0 statistical software (SPSS Inc, Chicago, IL, USA).

\section{RESULTS}

We created the Portuguese version of NOSE (NOSE-p) after cross-cultural adaptation and translation of the original version with acceptance of Stewart, the author of the original questionnaire (Figure 1).

The study population consisted of 33 patients, of which 19 
Table 1. Inter-item e item-total correlations.

\begin{tabular}{|c|c|c|c|c|c|}
\hline & Nasal congestion & Nasal obstruction & $\begin{array}{l}\text { Trouble breathing } \\
\text { through my nose }\end{array}$ & $\begin{array}{l}\text { Trouble } \\
\text { sleeping }\end{array}$ & $\begin{array}{l}\text { Unable to get enough air } \\
\text { through my nose during } \\
\text { exercise or exertion }\end{array}$ \\
\hline \multicolumn{6}{|l|}{ Nasal congestion } \\
\hline Nasal obstruction & 0,644 & & & & \\
\hline Trouble breathing through my nose & 0,547 & 0,659 & & & \\
\hline Trouble sleeping & 0,492 & 0,330 & 0,455 & & \\
\hline $\begin{array}{l}\text { Unable to get enough air through my nose dur- } \\
\text { ing exercise or exertion }\end{array}$ & 0,296 & 0,419 & 0,480 & 0,448 & \\
\hline NOSE total & 0,787 & 0,773 & 0,797 & 0,758 & 0,689 \\
\hline
\end{tabular}

were male $(57.6 \%)$ and 14 were female $(42.4 \%)$. The average age was $39.3 \pm 11.9$ (21-65) years (mean \pm SD). All patients were evaluated preoperatively and three months after surgery.

The internal consistency of NOSE-p was adequate, with Cronbach's alpha of 0.807 . Inter-items and item-total correlations are reported on Table 1. Analysis of inter-item and item-total correlations demonstrated expected associations, consistent with the prior English-language validation ${ }^{(6)}$, and demonstrated that the instrument represents a single unified construct, but not all individual items are measuring the exact same concepts. Items 'nasal congestion or stuffiness,' 'nasal blockage or obstruction' and 'trouble breathing through my nose' had a significant correlation coefficient with each other. Items 'trouble sleeping' and 'unable to get enough air through my nose during exercise or exertion' also had a significant correlation coefficient with all items, except for the correlation coefficient between 'nasal blockage or obstruction' and "trouble sleeping" $(\mathrm{r}=0.330)$, and 'nasal congestion or stuffiness' and 'Unable to get enough air through my nose during exercise or exertion' $(r=0.296)$. All items had an adequate correlation with subscale total.

Test-retest reliability was calculated using the GoodmanKruskal gamma coefficient in 29 patients. The reliability coefficient was very good, with gamma $=0.776(p<0.001)$.

In addition to face validity from the translation process and prior English-language validation, the instrument showed excellent between-groups discrimination. Study patients had a mean rank of 33.0, and the group of non-rhinologic patients had a mean rank of 8.5 (Mann-Whitney U-test, p $<0.001$ ).

The instrument was also very responsive, with a calculated standardized response mean (SRM) of 1.66, and effect size (ES) of 2.72 .

\section{DISCUSSION}

This study completed the process of cross-cultural adaptation and validation to the Portuguese language of a disease-specific health status questionnaire to evaluate nasal obstruction. The procedures of translation and cross-cultural adaptation were completed without difficulty, and produced an intelligible
Portuguese version of the NOSE questionnaire. The process of cross-cultural adaptation ensures that item content was maintained. The participation of multiple translators and a forward and backward translation are all important steps. A poor translation process may lead to an instrument that is not equivalent to the original questionnaire and the lack of equivalence limits the comparability of responsiveness across populations ${ }^{(9)}$ and it warrants the application of the questionnaire only for group level comparisons, rather than individual level comparison.

The NOSE-p instrument demonstrated good psychometric properties. Internal consistency reliability, test-retest reliability, and validity were good, and the response sensitivity to change was very high. These findings are consistent with the original English-language validation by Stewart et al ${ }^{(6)}$. The NOSE-p retains both the item-level characteristics, such as item-to-scale correlations and internal consistency, as well as the scorelevel characteristics, such as reliability, construct validity, and responsiveness, as the original English-language version. The NOSE-p instrument presents the advantage of being fast and simple to complete, with minimal burden to the patient. Although high inter-item correlation $(>0.7)$ could suggest there may be some redundancy of the item, data from original studies ${ }^{(6,10)}$ suggest that further studies with a larger sample size would elucidate this.

Although it is brief, it still has excellent responsiveness to change. Short questionnaires can have more sensibility for clinical modifications than lengthy ones. It is important to measure the actual responsive (i.e. using the standardized response mean), because it is a better predictor of responsiveness than internal consistency reliability, as shown by Puhan et al ${ }^{(11)}$.

There are other validated instruments available to evaluate nasal complaints ${ }^{(12)}$. These include the Chronic Sinusitis Survey (CSS) ${ }^{(13)}$, the Rhinosinusitis Disability Index $(\mathrm{RSDI})^{(14)}$, the Sino-Nasal Outcome Test (SNOT-20) ${ }^{(15)}$, the Rhinoconjunctivitis Quality of Life Questionnaire (RQLQ) ${ }^{(16)}$, and the Allergy Outcome Survey (AOS) ${ }^{(17)}$. However, CSS, RSDI and SNOT-20 were designed to evaluate patients with chronic sinusitis, and not to evaluate solely nasal obstruction. 


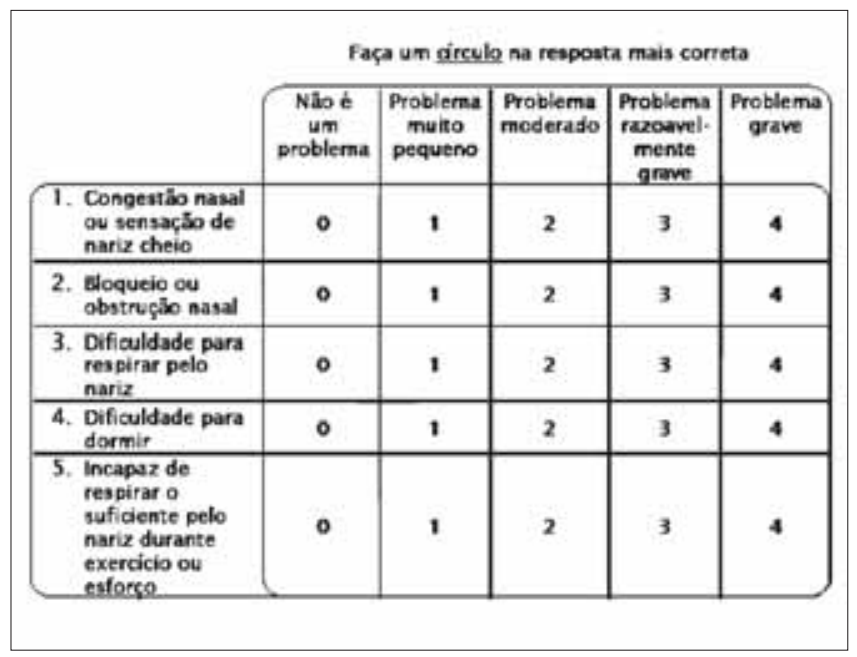

Figure 1. Final version of NOSE in portuguese language.

RQLQ and AOS were designed to evaluate patients with allergic rhinitis and conjunctivitis. Finally, SNOT-20 and AOS do not evaluate nasal obstruction. The NOSE instrument measures the patient's perception of nasal airway obstruction. This is very important because the patient's subjective perception of disease severity has, at best, a very weak association with objective assessments of severity, such as anatomic assessment or peak flow ${ }^{(18)}$. Disease-specific quality of life instruments apparently quantify an aspect of disease not detected by objective testing. The key point of the NOSE questionnaire is that it could evaluate nasal obstruction in any disease, not only in rhinitis or rhinosinusitis ${ }^{(12)}$.

As in the original study, the NOSE-p instrument was validated to evaluate groups of patients, and it was not designed to be used with individual patient data or to predict an outcome in individuals. The NOSE-p will be very useful in trials to evaluate not only outcomes in routine septoplasty surgery ${ }^{(10)}$, but also of other interventions, such as rhinoplasty ${ }^{(19)}$ and extracorporeal septoplasty ${ }^{(20)}$. Other surgical and non-surgical interventions can also be evaluated using the NOSE scale, for example radiofrequency ablation ${ }^{(21)}$ or topical nasal sprays.

A potential limitation of this is study was the consecutive convenience sampling used, which might not be representative of the entire population of Portuguese patients with nasal obstruction. A probability sample would be ideal, but more expensive and cumbersome.

In summary, the increasing interest in measures to assess the personal viewpoint of patients' health has led to an increased demand for reliable and valid standardized questionnaires of Health-Related Quality of Life ${ }^{(22)}$. It is very important to adapt appropriate instruments using a thorough process of cross-cultural adaptation and validation, to have quality of life questionnaires for use in clinical studies.

In conclusion, the NOSE was successfully adapted to the Portuguese language and the translated version proved to have adequate psychometric properties. NOSE-p will be a valuable tool for assessing outcome in clinical trials. This is the first adaptation of NOSE into a non-English language. Further studies will be required to examine the correlation between the NOSE-p and general/generic health status instruments.

\section{ACKNOWLEDGEMENTS}

This research was conducted with the support of Brazilian Association of Otolaryngology-Head and Neck Surgery (ABORLCCF, Brazil). Thiago F.P. Bezerra is supported by National Research Council (CNPQ, Brazil). Richard L. Voegels is supported by National Research Council (CNPQ, Brazil) and Sao Paulo Research Foundation (FAPESP, Brazil). The authors would like to thank Lucia Rocha for especial help with the cross-cultural adaptation process and the staff from the Clinics Hospital. The patients who agreed to participate in this study deserve our deeply recognition.

\section{AUTHORSHIP CONTRIBUTION}

TFPB: conception and design; acquisition, analysis and interpretation of data; drafting of the manuscript. FGMP: conception and design; interpretation of data and critical revision of the manuscript. RMMP: conception and design; MGS: conception and design; critical revision of the manuscript. RLV: conception and design; interpretation of data and critical revision of the manuscript

All authors have given their final approval of this version of the manuscript to be published.

\section{CONFLICT OF INTEREST}

Michael G. Stewart is medical advisory board member for Schering-Plough.

\section{REFERENCES}

1. Jessen M, Malm L. Definition, prevalence and development of nasal obstruction. Allergy 1997; 52 (40 Suppl): 3-6.

2. Pirila T, Tikanto J. Unilateral and bilateral effects of nasal septum surgery demonstrated with acoustic rhinometry, rhinomanometry, and subjective assessment. Am J Rhinol 2001; 15: 127-133.

3. Siegel NS, Gliklich RE, Taghizadeh F, et al. Outcomes of septoplasty. Otolaryngol Head Neck Surg. 2000; 122: 228-232.

4. Samad I, Stevens HE, Maloney A. The efficacy of nasal septal surgery. J Otolaryngol. 1992; 21: 88-91.

5. Arunachalam PS, Kitcher E, Gray J, et al. Nasal septal surgery: evaluation of symptomatic and general health outcomes. Clin Otolaryngol Allied Sci. 2001; 26: 367-370.

6. Stewart MG, Witsell DL, Smith TL, et al. Development and validation of the Nasal Obstruction Symptom Evaluation (NOSE) scale. Otolaryngol Head Neck Surg. 2004; 130: 157-163.

7. Fokkens W, Lund V, Mullol J. EP3OS 2007: European position paper on rhinosinusitis and nasal polyps 2007. A summary for otorhinolaryngologists. Rhinology 2007; 45: 97-101.

8. Boynton PM, Greenhalgh T. Selecting, designing, and developing your questionnaire. BMJ (Clinical research ed.). 2004; 328: 13121315.

9. Beaton DE, Bombardier C, Guillemin F, et al. Guidelines for the process of cross-cultural adaptation of self-report measures. Spine. 2000; 25: 3186-3191.

10. Stewart MG, Smith TL, Weaver EM, et al. Outcomes after nasal septoplasty: results from the Nasal Obstruction Septoplasty Effectiveness (NOSE) study. Otolaryngol Head Neck Surg. 2004; 130: $283-290$. 
11. Puhan MA, Bryant D, Guyatt GH, et al. Internal consistency reliability is a poor predictor of responsiveness. Health Qual Life Outcomes. 2005; 3: 33

12. van Oene CM, van Reij EJ, Sprangers MA, et al. Qualityassessment of disease-specific quality of life questionnaires for rhinitis and rhinosinusitis: a systematic review. Allergy 2007; 62: 1359-1371.

13. Gliklich RE, Metson R. Techniques for outcomes research in chronic sinusitis. Laryngoscope 1995; 105 (4 Pt 1): 387-390.

14. Benninger MS, Senior BA. The development of the Rhinosinusitis Disability Index. Arch Otolaryngol Head Neck Surg. 1997; 123: 1175-1179.

15. Piccirillo JF, Merritt MG, Jr., Richards ML. Psychometric and clinimetric validity of the 20-Item Sino-Nasal Outcome Test (SNOT-20). Otolaryngol Head Neck Surg. 2002; 126: 41-47.

16. Juniper EF, Guyatt GH. Development and testing of a new measure of health status for clinical trials in rhinoconjunctivitis. Clin Exp Allergy. 1991; 21: 77-83.

17. Kemker BJ, Corey JP, Branca J, et al. Development of the allergy outcome survey for allergic rhinitis. Otolaryngol Head Neck Surg. 1999; 121: 603-605.

18. Stewart MG, Smith TL. Objective versus subjective outcomes assessment in rhinology. Am J Rhinol. 2005; 19: 529-535.

19. Rhee JS, Poetker DM, Smith TL, et al. Nasal valve surgery improves disease-specific quality of life. Laryngoscope. 2005; 115: 437-440.
20. Most SP. Analysis of outcomes after functional rhinoplasty using a disease-specific quality-of-life instrument. Arch Facial Plast Surg. 2006; 8: 306-309.

21. Harrill WC, Pillsbury HC, 3rd, McGuirt WF, et al. Radiofrequency turbinate reduction: a NOSE evaluation. Laryngoscope. 2007; 117: 1912-1919.

22. Badia X, Webb SM, Prieto L, Lara N. Acromegaly Quality of Life Questionnaire (AcroQoL). Health Qual Life outcomes. 2004; 2 : 13 .

Thiago F.P. Bezerra

Department of Otorhinolaryngology and Ophthalmology

School of Medicine

University of São Paulo

São Paulo

Brazil

Tel: +55-11-3069 7834

E-mail: bezerratfp@gmail.com

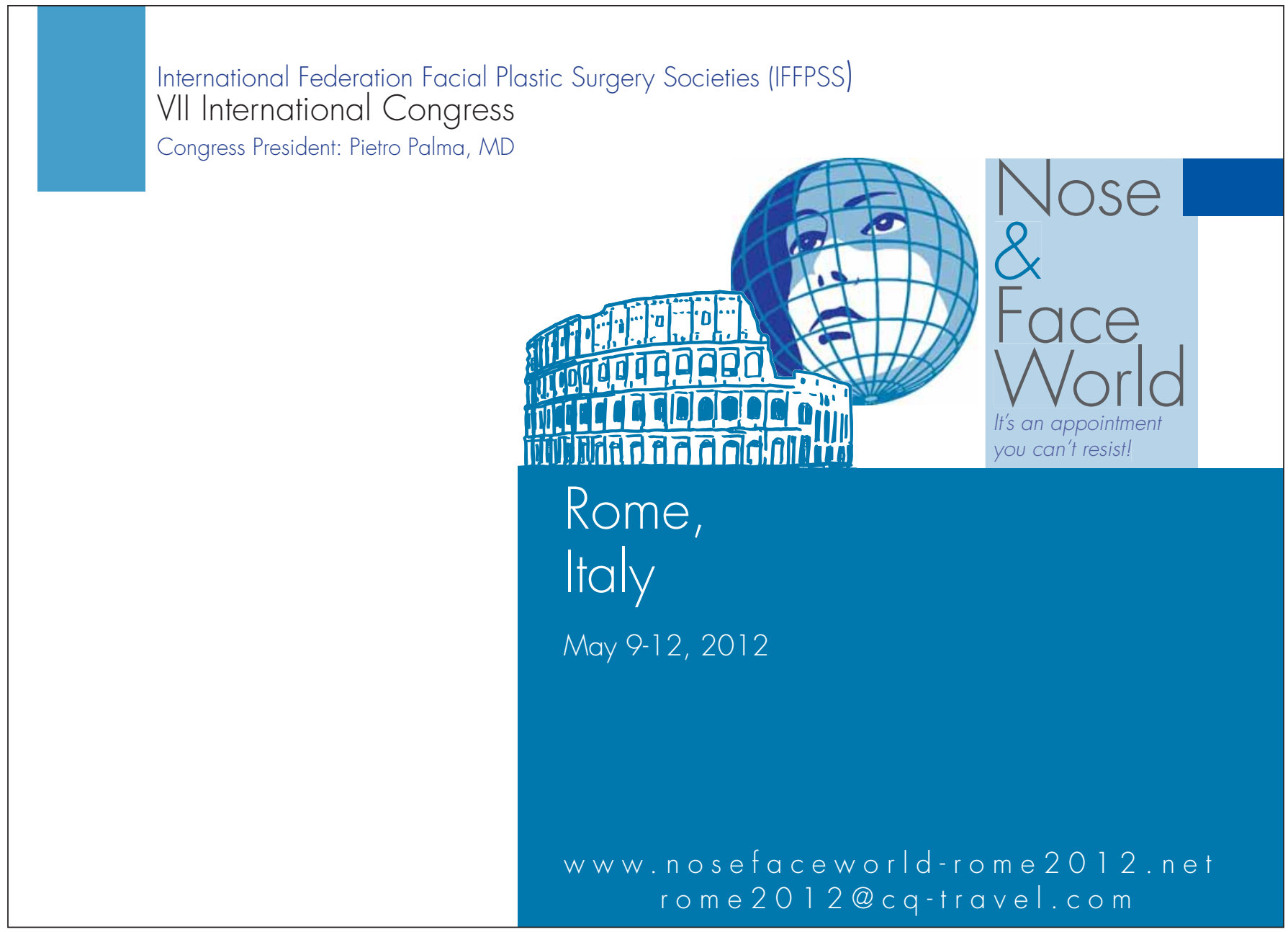

